\title{
Gallium self-diffusion in gallium phosphide
}

\author{
Lei Wang, J. A. Wolk, L. Hsu, and E. E. Haller ${ }^{\mathrm{a})}$ \\ University of California at Berkeley and Lawrence Berkeley National Laboratory, Berkeley, \\ California 94720
}

J. W. Erickson

Charles Evans \& Associates, Redwood City, California 94063

M. Cardona and T. Ruf

Max-Planck-Institut für Festkörperforschung, Heisenbergstrasse 1, D-70569 Stuttgart, Germany

\section{J. P. Silveira and F Briones}

Instituto de Microelectrónica de Madrid, Centro Nacional de Microelectrónica, CSIC, Serrano 144, 28066 Madrid, Spain

(Received 27 December 1996; accepted for publication 3 February 1997)

\begin{abstract}
Ga self-diffusion in gallium phosphide $(\mathrm{GaP})$ is measured directly in isotopically controlled $\mathrm{GaP}$ heterostructures. Secondary ion mass spectroscopy (SIMS) is used to monitor intermixing of ${ }^{69} \mathrm{Ga}$ and ${ }^{71} \mathrm{Ga}$ between isotopically pure $\mathrm{GaP}$ epilayers which are grown by molecular beam epitaxy (MBE) on GaP substrates. The Ga self-diffusion coefficient in undoped GaP is determined to be $D=2.0 \mathrm{~cm}^{2} \mathrm{~s}^{-1} \exp \left(-4.5 \mathrm{eV} / k_{\mathrm{B}} T\right)$ between 1000 and $1190{ }^{\circ} \mathrm{C}$ under phosphorus-rich condition. The self-diffusion entropy is found to be $\sim 4 k_{B}$. (C) 1997 American Institute of Physics. [S0003-6951(97)00914-5]
\end{abstract}

Self-diffusion is the migration of constituent atoms in materials. This process is mediated by native defects in solids and thus can be used to study the dynamics and kinetics of these defects. The knowledge obtained in these studies is pivotal for the understanding of many important mass transport processes such as impurity diffusion in semiconductors. There has been a great deal of research on self-diffusion processes in silicon, germanium, and many compound semiconductors. $^{1,2}$ Diffusion of column-III atoms in III-V compounds is of special interest because it affects the stability of epitaxial structures, such as those in GaAs/AlGaAs lasers and transistors. ${ }^{3} \mathrm{Ga}$ self-diffusion in GaAs and AlGaAs has been extensively investigated. ${ }^{4}$ A popular model for the process in intrinsic and $n$-type materials assumes the triply negatively charged Ga vacancy to be the primary native defect mediating $\mathrm{Ga}$ migration, with an activation enthalpy of $6 \mathrm{eV} .^{4}$ Although it can explain most experimental observations, the model has not put to rest the controversy surrounding the mechanism and characteristics of the process. For instance, our recent study using an isotopically controlled GaAs epitaxial structure yielded an activation enthalpy of $4.24 \mathrm{eV} .^{5}$ The topic of self-diffusion in compound semiconductors clearly warrants further investigation. Expanding the research on $\mathrm{Ga}$ self-diffusion beyond GaAs to other III-V compound semiconductors can be quite instructive in elucidating the microscopic mechanism. This motivated us to carry out an investigation on Ga self-diffusion in gallium phosphide $(\mathrm{GaP})$ using the same method as employed in Ref. 5. To our knowledge, the self-diffusion process has never been directly studied in this semiconductor.

For the experiment, ${ }^{71} \mathrm{GaP}$ and ${ }^{69} \mathrm{GaP}$ epitaxial layers $200 \mathrm{~nm}$ thick were grown by solid source molecular beam epitaxy (MBE) at $700{ }^{\circ} \mathrm{C}$ on undoped GaP substrates. The natural $\mathrm{Ga}$ isotope composition in the $\mathrm{GaP}$ substrates is $60.2 \%{ }^{69} \mathrm{Ga}$ and $39.8 \%{ }^{71} \mathrm{Ga}$. The compositions in the isoto-

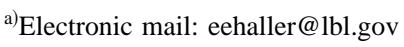

pically controlled epilayers, on the other hand, are $99.6 \%$ ${ }^{69} \mathrm{Ga}\left({ }^{71} \mathrm{Ga}\right)$ and $0.4 \%{ }^{71} \mathrm{Ga}\left({ }^{69} \mathrm{Ga}\right)$. Figure 1 displays the concentration profiles of the $\mathrm{Ga}$ isotopes in as-grown samples measured by secondary ion mass spectroscopy (SIMS). In the SIMS measurement, the primary ion beam was formed with $5.5-\mathrm{keV} \mathrm{Cs}^{+}$ions. $\mathrm{GaCs}^{+}$molecules were detected as secondary species as the sputtering proceeded. A resolution of $3.5 \mathrm{~nm}$ per decade in Ga concentration can be achieved for an ascending concentration step at a sharp interface (Fig. 1). The broadening of the interface is primarily due to ion mixing and surface roughening by energetic $\mathrm{Cs}^{+}$ ions.

Before thermal annealing, the samples were cleaned with solvents, $\mathrm{HCl}$, and $\mathrm{HF}$. They were sealed in quartz ampoules which were evacuated to $\sim 10^{-6}$ Torr. To preserve the

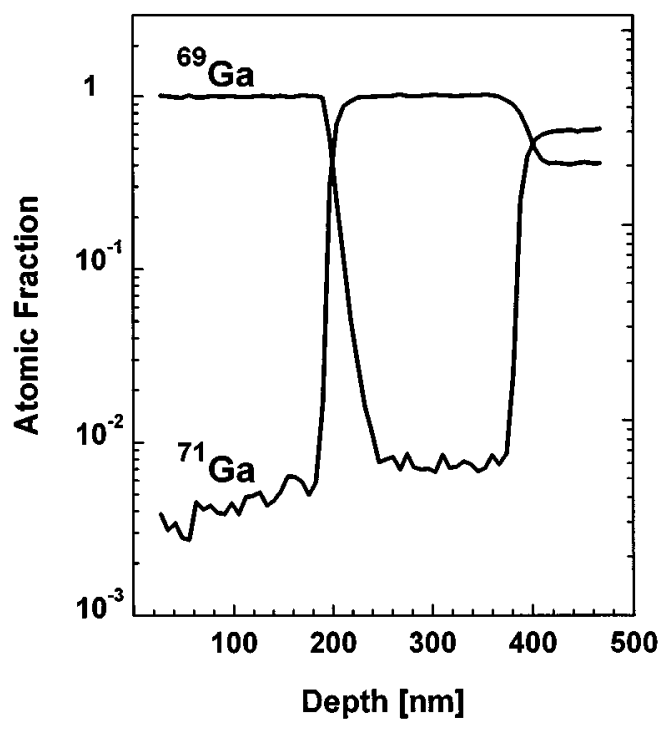

FIG. 1. SIMS depth profiles of ${ }^{69} \mathrm{Ga}$ and ${ }^{71} \mathrm{Ga}$ in as-grown $\mathrm{GaP}$ isotope epilayers on a natural $\mathrm{GaP}$ substrate. 


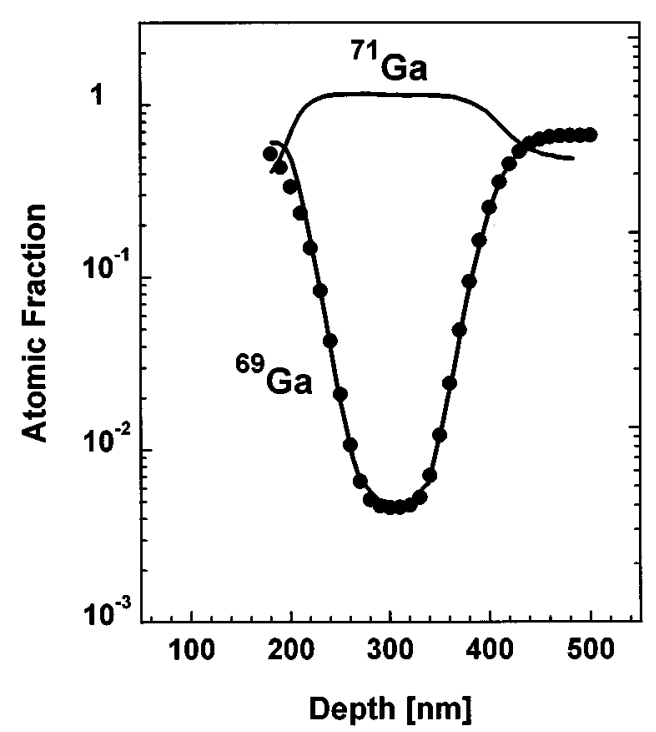

FIG. 2. SIMS depth profiles of ${ }^{69} \mathrm{Ga}$ and ${ }^{71} \mathrm{Ga}$ in $\mathrm{GaP}$ isotope epilayers annealed at $1111^{\circ} \mathrm{C}$ for $231 \mathrm{~min}$. The filled circles represent the calculated ${ }^{69} \mathrm{Ga}$ concentration profile.

sample surfaces, pure red phosphorus $(99.99 \%)$ was placed in the ampoules so as to create a phosphorus overpressure of $\sim 1 \mathrm{~atm}$ at each annealing temperature, assuming the ideal gas law for $\mathrm{P}_{4}$. In addition the samples were buried in $\mathrm{GaP}$ powder. Annealing temperatures were controlled to within $\pm 1{ }^{\circ} \mathrm{C}$ and the ampoules were rapidly quenched in water following annealing.

In our experiment, Ga self-diffusion is studied by monitoring the intermixing of the $\mathrm{Ga}$ isotopes between the two epilayers or between the substrate and the adjacent epilayer. Since the two Ga isotopes undergo significant intermixing during the heat treatment, the SIMS broadening effects can be neglected when analyzing concentration profiles of the $\mathrm{Ga}$ isotopes in annealed samples. Assuming Fick's equations describe the self-diffusion process and the diffusion coefficient $D$ is constant, ${ }^{6}$ the concentrations of the Ga isotopes can be expressed as

$$
C(x)=\frac{C_{1}+C_{2}}{2}-\frac{C_{1}-C_{2}}{2} \operatorname{erf}\left(\frac{x}{R}\right),
$$

where $x=0$ at the epitaxial interface, $C_{1}$ and $C_{2}$ are the initial $\mathrm{Ga}$ isotope concentrations at the left- and right-hand side of the interface, respectively, and $\operatorname{erf}(y)$ is the error function. The characteristic diffusion length $R$ is defined as

$$
R=2 \sqrt{D t},
$$

where $D$ is the Ga self-diffusion coefficient and $t$ is the annealing time.

The SIMS data can then be compared with calculated values of $C(x)$. Adjusting the diffusion length $R$, a fit of $C(x)$ to the SIMS profile can be made. Figure 2 shows the SIMS profiles (solid lines) and the calculated $C(x)$ of ${ }^{69} \mathrm{Ga}$ (circles) and ${ }^{71} \mathrm{Ga}$ (continuous line) in a sample annealed at $T=1111^{\circ} \mathrm{C}$ for $3 \mathrm{~h}$ and $51 \mathrm{~min}$. Excellent agreement is obtained between the measured and the calculated profiles over two and a half orders of magnitude in concentration. This

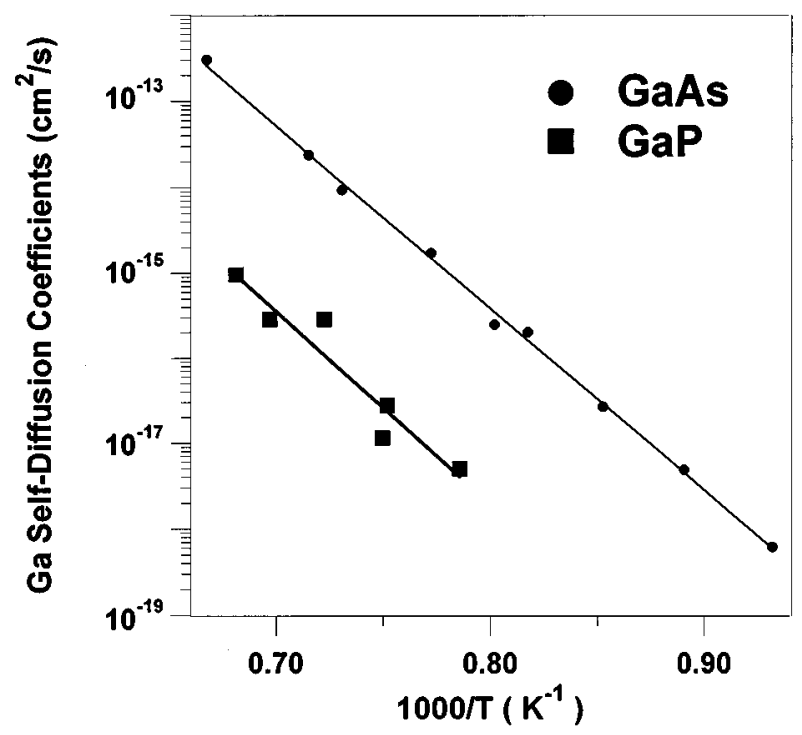

FIG. 3. Arrhenius plots of Ga self-diffusion coefficients in GaAs (filled circles) and $\mathrm{GaP}$ (filled squares).

agreement strongly supports the assumptions made in Eq. (1). The fitting procedure leads to an accurate determination of $D$ through Eq. (2).

In Fig. 3, $D$ is plotted versus temperature $T$. The $D$ values span over two orders of magnitude in the temperature range from 1000 to $1190{ }^{\circ} \mathrm{C}$. They can be represented by

$$
D=D_{0} \exp \left(-\frac{H^{S D}}{k_{\mathrm{B}} T}\right)
$$

where the activation enthalpy $H^{\mathrm{SD}}$ and the pre-exponential factor $D_{0}$ are determined to be $4.5 \mathrm{eV}$ and $2.0 \mathrm{~cm}^{2} \mathrm{~s}^{-1}$, respectively. The self-diffusion entropy $S^{S D}$ (Ref. 2) can be estimated from $D_{0}$ with

$$
D_{0}=g a^{2} v_{0} \exp \left(\frac{S^{S D}}{k_{\mathrm{B}}}\right),
$$

where $g$ is a geometric factor of the crystal structure, $a$ the lattice constant, $v_{0}$ the frequency of the highest vibrational mode in the crystal, and $k_{\mathrm{B}}$ the Boltzmann constant. In GaP, $g \sim 1, a=5.45 \AA$, and $v_{0}=1.2 \times 10^{13} \mathrm{~Hz}$. Using these values we obtain $S^{S D}=4 k_{\mathrm{B}}$.

The Ga self-diffusion coefficients in GaAs from our previous work ${ }^{5}$ are also shown in Fig. 3 for comparison. The activation enthalpy and entropy for GaAs are $4.24 \mathrm{eV}$ and $7.5 k_{\mathrm{B}}$, respectively.

As in the case of GaAs, the Ga self-diffusion coefficients in $\mathrm{GaP}$ follow an Arrhenius relation described by Eq. (3), indicating that a single type of native defect is most likely responsible for mediating the Ga self-diffusion in GaP over our temperature range. In intrinsic GaAs, the defect mediating Ga self-diffusion has been ascertained to be the triply negatively charged gallium vacancy acceptor, $V_{\mathrm{Ga}}^{3-} \cdot{ }^{4} \mathrm{In}$ view of our limited data range, such an assignment may still be premature for GaP. From a recent positron annihilation study in GaP, Krause-Rehberg et al. ${ }^{7}$ reported that positron trapping by vacancies behaves similarly in $\mathrm{GaP}$ as in GaAs. Vacancies are detected by these authors only in $n$-type $\mathrm{GaP}$ at 
room temperature, with a detection limit of $2 \times 10^{15} \mathrm{~cm}^{-3}$. These findings neither support nor exclude the possibility that in intrinsic $\mathrm{GaP}$ it is also the acceptor-like $V_{\mathrm{Ga}}$ that mediates Ga self-diffusion.

Figure 3 shows that the Ga self-diffusion coefficient in $\mathrm{GaP}$ is about two orders of magnitude lower than that in GaAs. The decreased cation diffusion in III-V compounds, with phosphorus replacing arsenic as anion, has been previously observed. ${ }^{8,9}$ Interdiffusion of $\mathrm{Ga}$ and $\mathrm{Al}$ was determined to be two orders of magnitude slower in the AlGaInP/ GaInP superlattice system than in the $\mathrm{AlGaAs} / \mathrm{GaAs}$ system. The change has been attributed to the stronger $\mathrm{Ga}-\mathrm{P}$ bond compared to the Ga-As bond. ${ }^{9}$ Although we measured a higher activation enthalpy in $\mathrm{GaP}(4.5 \mathrm{eV})$ than in GaAs $(4.24 \mathrm{eV})$, the difference is not large enough to be outside the experimental uncertainty. More reliable is the difference in the pre-exponential factor $D_{0}$, or the entropy term $S^{S D}$, between $\mathrm{GaP}\left(4 k_{\mathrm{B}}\right)$ and $\mathrm{GaAs}\left(7.5 k_{\mathrm{B}}\right)$. This entropy term is the sum of the formation entropy $S^{F}$ and migration entropy $S^{M}$ for the native defect mediating the self-diffusion. $S^{F}$ or $S^{M}$ represents the number of equivalent formation configurations or migration jumps. The significant difference in $S^{S D}$ indicates profound variations in the way that the mediating native defects are formed or migrate in $\mathrm{GaP}$ as compared to GaAs. The small value of $S$ supports a simple native defect species as the major diffusion vehicle.

Our determination of the activation enthalpy and entropy for the Ga self-diffusion is important as a first step in understanding the process. To determine the native defect which is responsible for the self-diffusion process, further studies, including effects of doping and stoichiometry, are required.
To summarize, we have studied Ga self-diffusion in undoped GaP isotope heterostructures under P-rich conditions. It is concluded that the self-diffusion process is most likely mediated by one type of a simple native defect. The process is characterized by an activation enthalpy of $4.5 \mathrm{eV}$ and an entropy term of $4 k_{\mathrm{B}}$. In comparison with Ga self-diffusion in GaAs, the significantly smaller entropy term in GaP raises a question as to what causes this difference. The excellent success of molecular dynamics calculations for $H$ and $S$ for GaAs self-diffusion ${ }^{10}$ will hopefully be an incentive to perform similar calculations for GaP.

This work is supported in part by the U.S. NSF Grant DMR-94 11763 and in part by the Director, Office of Energy Research, Office of Basic Energy Sciences, Materials Sciences Division of the U.S. Department of Energy under Contract No. DE-AC03-76SF00098.

${ }^{1}$ W. Frank, U. M. Gösele, H. Mehrer, and A. Seeger, Diffusion in Crystalline Solids (Academic, New York, 1984), p. 63.

${ }^{2}$ A. F. W. Willoughby, Mater. Res. Soc. Symp. Proc. 14, 237 (1983).

${ }^{3}$ D. G. Deppe and N. Holonyak, Jr., J. Appl. Phys. 64, R93 (1988).

${ }^{4}$ T. Y. Tan, U. M Gösele, and S. Yu, Crit. Rev. Solid State Phys. 17, 47 (1991).

${ }^{5}$ L. Wang, L. Hsu, E. E. Haller, J. W. Erickson, A. Fischer, K. Eberl, and M. Cardona, Phys. Rev. Lett. 76, 2342 (1996).

${ }^{6} \mathrm{~J}$. Crank, The Mathematics of Diffusion, 2nd ed. (Clarendon, Oxford, 1993).

${ }^{7}$ R. Krause-Rehberg, A. Polity, W. Siegel, and G. Kühnel, Semicond. Sci. Technol. 8, 290 (1993).

${ }^{8}$ S. O'Brien, D. P. Bour, and J. R. Shealy, Appl. Phys. Lett. 53, 1859 (1988).

${ }^{9}$ K. J. Beernink, D. Sun, D. W. Treat, and B. P. Bour, Appl. Phys. Lett. 66, 3597 (1995)

${ }^{10}$ M. Bockstedte and M. Scheffler, Bull. Am. Phys. Soc. Ser. II 41, 562 (1996). 